\title{
ECOCOMPATIBILITÉ DES DÉCHETS : VERS UNE PRISE EN COMPTE DE LA NOTION D'IMPACT POUR L'ÉLIMINATION ET LA VALORISATION DES DÉCHETS
}

\author{
Valérie Mayeux*, Yves Perrodin** \\ Ademe, Centre d'Angers, Insa de Lyon, Division Polden
}

Les conditions de stockage ou de valorisation des déchets sont aujourd'hui encore définies sur des bases réglementaires et techniques reposant sur la mise en place de la meilleure technologie disponible et économiquement acceptable, ne reposant que très peu sur une notion d'impact sur les milieux d'accueil sollicités.

Le présent article décrit le principe et les premiers résultats d'un programme de recherche de 3 ans, lancé à l'initiative de l'Ademe, baptisé « Programme Écocompatibilité " et visant à intégrer progressivement une logique d'impact dans la gestion des déchets.

L'évaluation de l'écocompatibilité des déchets est basée sur la comparaison des flux de polluants émis par les déchets (pris dans un scénario donné) et des flux de polluants jugés acceptables pour les différents milieux récepteurs (aquatiques et « sol ») concernés.

Le premier travail réalisé par les équipes participant au programme a consisté à identifier et hiérarchiser les nombreux facteurs d'influence et paramètres de caractérisation à prendre en compte pour ces évaluations.

Un programme d'expérimentations à réaliser au laboratoire et à grande échelle lors des deuxième et troisième années du programme a ensuite été mis en place.

\section{PRÉAMBULE HISTORIQUE : DE LA} DÉCHARGE AU CENTRE DE STOCKAGE DES DÉCHETS ULTIMES STABILISÉS ET À LA VALORISATION

\section{5 : Première loi sur les déchets}

Avec la loi de 1975 sur les déchets et celle de 1976 sur les installations classées, la France se dote d'un système législatif performant, basé sur le principe de responsabilité du producteur de déchet et sur une "logique installation classée » pour gérer au mieux ses déchets, de leur production à leur élimination.

Son caractère dynamisant contribue au développement de nombreux centres de traitement, qu'il s'agisse de centres col-
Disposal conditions or re-use of waste are still defined on regulation and technical basis depending on the best available and economically acceptable technology. Current regulations take into account the notion of impact on the environmental receptors only to a very limited extent.

This article describes the principle and the first results of a three year research program, initiated by the French Agency of Energy and Environment (Ademe), called « Ecocompatibility program » aiming to introduce progressively a logic of impact in the waste management.

The assessement of the waste ecocompatibility is based on the comparison of the flow of the waste pollutants (in a specified scenario) and the flow of pollutants considered as acceptable by the different environmental receptors (water or soil) concerned.

The first work conducted by the teams involved in this program was to identify and hierarchize the numerous factors of influence and the parameters of characterization to be considered for this assessement. An experimental program of tests to be performed at the labscale and on a full scale during the second an the third year of the elaborated program.

lectifs ou d'unités propres à des grands groupes industriels. Bien vite, un réseau complet d'installations collectives de traitement de déchets industriels se met en place : incinérateurs, unités de traitement physico-chimiques, décharges contrôlées, ...

Jugées pesantes au départ, les nouvelles mesures réglementaires contribuent au développement d'une nouvelle industrie dont les efforts vont croissants.

1992 : De nouvelles orientations pour le système réglementaire français dans le domaine des déchets

Le I 3 juillet 1992, la loi de 1975 fait peau neuve et instaure de nouveaux principes pour la gestion des déchets.

Si la notion de déchets ultimes ${ }^{2}$ révolutionne le monde des 
déchets, d'autres ont également leur importance. II s'agit : - de la prévention ou de la réduction de la production et de la nocivité des déchets en agissant sur la fabrication et sur la distribution des produits,

- de la valorisation des déchets par réemploi, recyclage ou tout autre action visant à obtenir à partir des déchets des matériaux réutilisables ou de l'énergie,

- de l'organisation du transport des déchets et de sa limitation tant en distance qu'en volume.

Le 18 décembre de cette même année, les arrêtés relatifs au stockage de certains déchets spéciaux ultimes et stabilisés confortent pour les déchets industriels les grands principes de la loi du 13 juillet et modifient sensiblement leurs conditions de stockage. Désormais, le stockage des déchets spéciaux implique la mise en place de plusieurs niveaux de sécurité :

- une sécurité passive : la géologie du site,

- trois niveaux de sécurité active :

- le drainage des eaux qui rentrent en contact avec le déchet afin de garantir leur élimination rapide vers le fond des alvéoles du stockage et leur évacuation hors des alvéoles, associé à un dispositif complet d'étanchéité,

- une couverture finale multicouches mise en place en fin d'exploitation,

- une sélectivité accrue des déchets admissibles, correspondant notamment à l'admission des seuls déchets ultimes et stabilisés.

\section{4 : Circulaire sur la valorisation des} mâchefers en technique routière

Le 9 mai 1994, une circulaire du ministère de l'Environnement précise les conditions de valorisation des mâchefers d'incinération des ordures ménagères. Elle définit en particulier trois types de mâchefers selon leurs comportements au test de lixiviation normalisé NF $\times 31-210$ :

- les mâchefers « $V$ » à faible fraction lixiviable, pouvant être valorisés en technique routière sous certaines conditions,

- les mâchefers « $M$ », intermédiaires, pouvant faire l'objet d'une maturation de 12 mois au plus (éventuellement complétée par un traitement de stabilisation) dans un centre de stockage temporaire en vue d'une valorisation,

- les mâchefers « $S$ » à forte fraction lixiviable devant être éliminés dans des centres de stockage permanent de déchets ménagers et assimilés dûment autorisés.

Le texte précise également que les instructions de la circulaire pourront être révisées en fonction des enseignements issus de travaux complémentaires en cours ou de l'expérience tirée de leur application.

\section{5-2000 : une évolution inévitable}

Les nouvelles conditions de stockage ou de valorisation des déchets sont encore définies sur des bases réglementaires et techniques reposant peu (ou pas) sur une notion d'impact sur les milieux d'accueil sollicités ; ceci notamment en raison du manque de bases scientifiques dans le domaine.

Ce défaut de bases scientifiques a le plus souvent été com- pensé par le développement de technologies améliorant toujours plus les conditions d'élimination des déchets.

Il apparaît aujourd'hui que cette approche de la meilleure technologie disponible ne peut plus constituer une réponse unique, en terme d'objectif et de niveaux à atteindre en matière de protection de l'environnement.

\section{UNE NOUVELLE CONCEPTION DE LA GESTION DES DÉCHETS}

\section{là ?}

\section{Logique d'impact : mais qu'entend-on par}

Travailler dans une logique d'impact consisterait non plus à bâtir les réglementations sur le respect de seuils fixés en fonction uniquement de la meilleure technologie disponible associée à un coût économique acceptable, mais à fonder les arbitrages (j'accepte ou je n'accepte pas) sur des outils fiables de mesures et d'évaluation des impacts réels du déchet sur l'environnement.

\section{Mais qu'est-ce que l'écocompatibilité ?}

L'écocompatibilité des déchets peut être définie comme une situation où les flux de polluants émis par les déchets placés dans un certain contexte physique, hydrogéologique et biophysico-chimique sont compatibles avec les flux de polluants acceptables par les milieux récepteurs concernés.

Cela ne veut pas dire pour autant que la définition du rejet écocompatible doive se traduire par une sollicitation maximum des milieux d'accueils, ni même que cette notion puisse privilégier l'usage de sites fortement pollués.

\section{MÉTHODOLOGIE D'ÉVALUATION DE L'ÉCOCOMPATIBILITÉ}

\section{Méthodologie globale}

II ressort de la définition établie ci-dessus que l'évaluation de l'écocompatibilité nécessite l'évaluation de trois termes majeurs :

- l'émission de flux polluants par le dépôt de déchet (ou l'ouvrage constitué à base de celui-ci), encore appelé terme source. Ce terme est lui-même fonction du potentiel polluant intrinsèque des déchets (appelé terme $A$ ) et du mode de disposition de ces déchets dans l'environnement (appelé terme $B$ ou terme enveloppe) ;

- le transport des flux de polluants de la sortie de l'enveloppe jusqu'aux milieux récepteurs, appelé terme $T$;

- le niveau d'acceptation des flux de polluants par les milieux récepteurs, appelé terme $C$.

Par ailleurs, compte tenu des déchets pour lesquels il est envisageable, dans un premier temps, d'arriver à une situation d'écocompatibilité (déchets stables à dominante minérale essentiellement), l'eau a été considérée comme le vecteur principal de transport des polluants du terme $A$ vers les milieux récepteurs.

Une présentation synthétique de cette approche méthodologique globale est donnée dans la figure $n^{\circ} \mathrm{I}$. 
Eau (pluie, ruissellement, infiltration...)

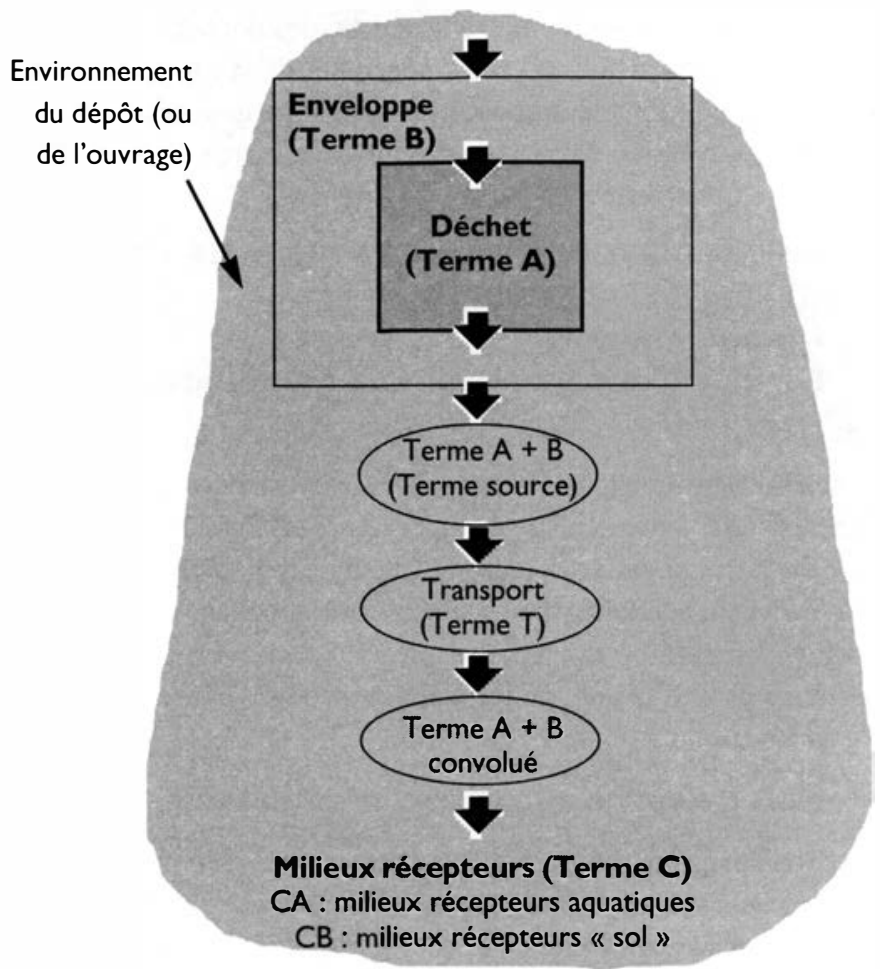

Définition de l'écocompatibilité : Soit $F A+B$ le flux de polluants émis par le couple $A+B, F(A+B)$ ' le flux de polluants atteignant le milieu récepteur après transport (encore appelé flux $A+B$ convolué), FcA le flux acceptable par le milieu récepteur aquatique, FCB le flux acceptable par le milieu récepteur sol. Le dépôt de déchets sera considéré comme écocompatible si $F(A+B)^{\prime}<F C A$ et $F(A+B)^{\prime}<F C B$

Figure I : Schéma global simplifié de l'évaluation de l'écocompatibilité des déchets

\section{Évaluation des différents termes de l'éco- compatibilité}

État des connaissances - Identification des facteurs d'influence et paramètres de caractérisation à prendre en compte pour l'évaluation

En 1995, afin d'acquérir les données nécessaires pour fixer la nature des travaux à mener sur des bases scientifiques cohérentes, l'Ademe, après sélection sur appel d'offres, a confié différentes études au groupement composé des équipes présentées dans l'organigramme (figure $n^{\circ} 2$ ).

Ces études ont portés sur :

- les modèles de comportement biophysicochimique des déchets,

- les outils de l'évaluation de l'écotoxicité,

- l'étude du terme enveloppe,

- les modèles de transport des solutions dans les milieux récepteurs,

- la caractérisation des effets des polluants sur les divers organismes vivants dans les milieux récepteurs « sol » (microorganismes, faune et flore) et aquatiques et leur utilisation comme indicateurs de la qualité des milieux concernés.

Ces études ont permis de répertorier les outils existants pour la mesure de chacun des termes nécessaires à l'évaluation de l'écocompatibilité des déchets, et de mettre en évidence les besoins de recherches complémentaires à effectuer. Elles ont notamment abouti à l'identification de l'ensemble des facteurs d'influence et paramètres de caractérisation à prendre en compte pour l'évaluation de l'écocompatibilité (voir tableau $\mathrm{n}^{\circ} \mathrm{I}$ ).

\section{Coordination générale Polden}

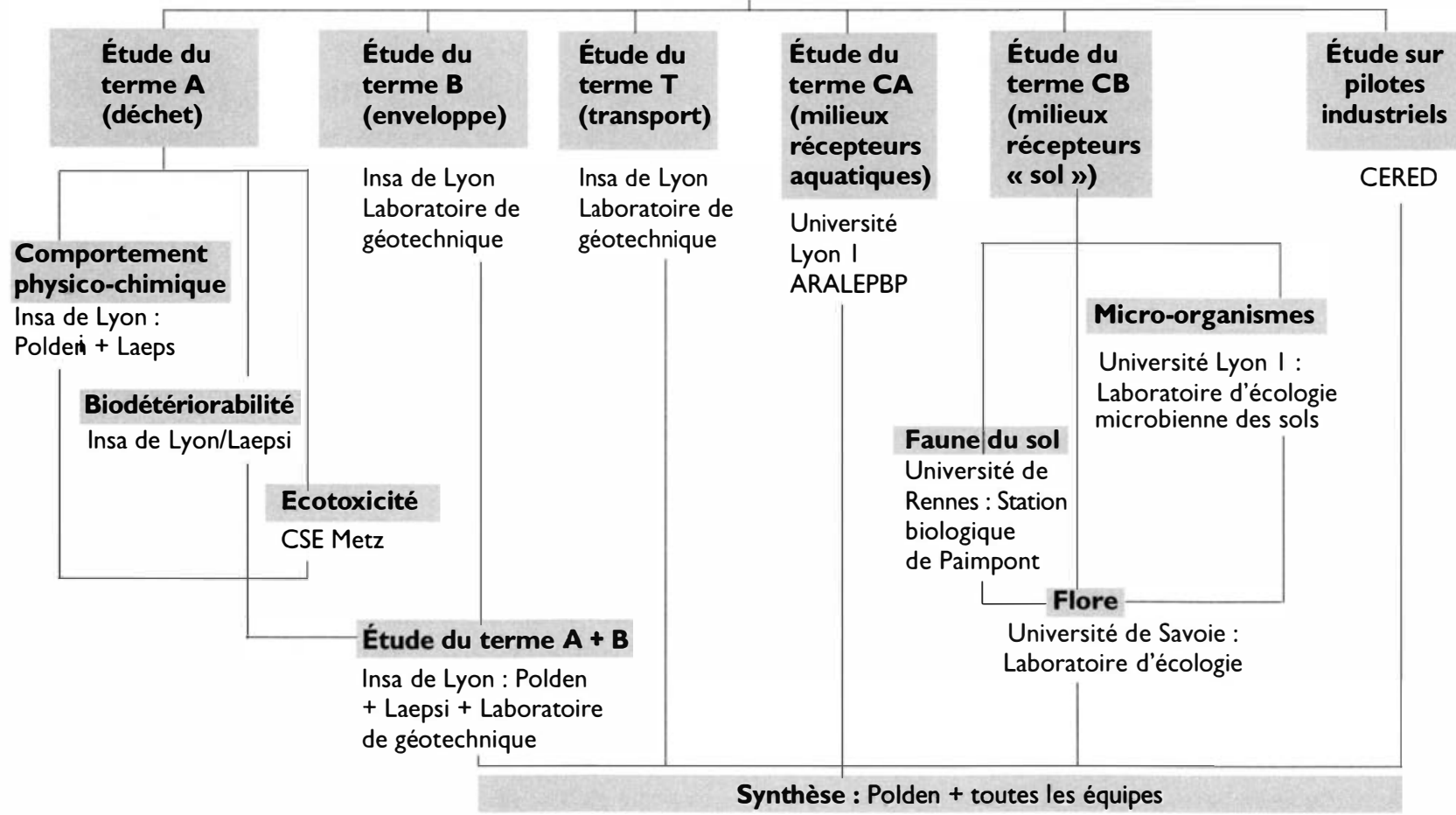

Figure 2 : Équipes de recherche participant au programme Ademe sur l'écocompatibilité des déchets 

pour l'évaluation de l'écocompatibilité des déchets

\section{Terme considéré}

Terme A Étude physico-chimique

tude biologique

Etude écotoxicologique
Terme B

\section{Facteurs d'influence}

- Structure du déchet (poreux, vitreux)

- État physique du déchet (granulaire, massif)

- Mode de contact principal de l'eau avec

le déchet (percolation, lessivage, immersion)

- Conditions climatiques (pluviométrie, cycles de températures, de gel/dégel et d'humidification/séchage,...)

- Nature chimique de la solution de lixiviation

- Nature du terme B

- Nature du milieu récepteur

- Conditions d'alimentation en eau et composition de la solution apportée,

- Température,

- pH, Eh, force ionique (salinité)

- Aération et/ou teneurs en oxygène dissous dans l'eau.

- Exposition à l'air,

- Exposition à la lumière,

- Apports de substances nutritives

- Exposition à un milieu naturel microbiologiquement actif.

- Facteurs relauifs aux conditions de l'essai : - nature, durée et fréquence de l'exposition, - facteurs physiques, chimiques et microbiologi ques susceptibles d'influer sur l'écotoxicité des déchets (photopériode, humidité, $\mathrm{pH}$ oxygène dissous, dureté, conductivité, salinité, contamination bactérienne,...)

- Facteurs relautfs aux organismes testés (type d'espèces utilisées, nombre d'espèces utilisées, santé des organismes testés, âge et stade de développement des organismes testés),

- Facteurs relatifs aux déchets (caracténisiques physico-chimiques et biologiques du terme A, caractéristiques du terme B)

- Conditions climatiques (pluviométrie, température, rayonnement solaire, vitesse du vent, variation de l'humidité,...) - Caractéristiques géologiques, hydrologiques, géotechniques et morphologiques du milieu récepteur

- Caractéristiques du déchet (physiques, mécaniques et de vieillissement)

- Mode de mise en place du déchet,

- Faune et flore présente,

- Aménagements extérieurs (dispositif de drainage amont,...)

Terme T

\section{Paramètres de caractérisation}

- Paramètres physico-chimiques (composition chimique, homogénéité, granulométrie, densité, porosité, perméabilié, humidité, $\mathrm{CAE}$, capacité de neutralisation, conductivité thermique) - Paramètres physico-chimiques mesurés sur l'eau en contact avec les déchets $(\mathrm{pH}$, potentiel Red-Ox, conductivité, concentrations en polluants)

- Paramètres mécaniques, structurels et de vieillissement (paramètres mécaniques, surface spécifique, résistance à la corrosion, résistance au gel/dégel, résistance à l'humidification/séchage, sensibilité à la carbonatation, taux de délitescence)

- Paramètres de comportement à la lixiviation:

- comportement à la lixiviation des broyés : contenu lixiviable, sensibilité aux caractéristiques du lixiviat ( $\mathrm{pH}$, potentiel Red-Ox, composition),

- comportement à la lixiviation des granulaires : percolation en colonne (noyée ou arrosée), sensibilité aux caractéristiques du lixiviant et au régime d'écoulement,

- comportement à la lixiviation des massifs : matrices poreuses (identification des paramètres du relargage diffusionnel, sensibilités aux caractéristiques du lixiviant), motrices vitreuses (vitesse initiale de dissolution, sensibilité au $\mathrm{pH}$, température et composition du lixiviant, altération hydrothermale du verre).

- teneur en eau ou activité en eau,

- capacité de rétention de l'eau,

- granulométrie, porosité,

- $\mathrm{pH}$, Eh, force ionique

- biomasse microbienne.

- présence et biodisponibilité de substances sources d'énergie pour les organismes chimiotrophes (CO, $\mathrm{S}, \mathrm{S}$ réduit, $\mathrm{NH}_{3}$, Ferll)

- biodégradabilité intrinsèque de la fraction organique du déchet.

- présence et biodisponibilité de composés accepteurs d'électrons dans le cas de milieux non aérés $\left(\mathrm{NO}_{3} ; \mathrm{SO}_{4}{ }^{-}, \mathrm{CO}_{2}\right.$ ou $\mathrm{HCO}_{3}$, Ferlll, $\left.\mathrm{MnIV}\right)$

- présence et biodisponibilité de nutriments $(P, \ldots)$

- présence de substances biocides ou biostatiques en quantité toxique.

- Effets globaux:

- survie,

- reproduction

- croissance,

- comportement,

- Effets spécifiques :

perturbation des mécanismes biochimiques,

- altération du patrimoine génétique,

- biomarqueurs

- Paramètres liés à la géométrie du dépôt (pente, surface, hauteur...)

- Paramètres liés à la structure de $B$ :

- couche végétale : épaisseur, type de végétation, capacité de rétention, teneur en eau

- couche filtrante : nature, dimensions, permittivité, porométrie, coefficient de perméabilité, ouverture de filtration, caractéristiques mécaniques (pour un géotextile : résistance au poinçonnement, à la traction et à la déchirure)

- couche de protection contre les biointrusions : nature, épaisseur

- couche drainante : nature, perméabilité, porométrie, caractéristiques mécaniques, pente

- couche étanche : nature, position par rapport au déchet, complexité des couches, épaisseur, perméabilité, état, paramètres de caractérisation du matériau

- Paramètres liés à la mise en œurre des déchets (état de surface, compactage, vitesse de remplissage du dépôt, ...)

- Pasmètres liés à la sabilité d'ensemble du dépôt : indice portant immédiat, cohésion, angle de frotternent...

- Conductivité hydraulique ou perméabilité

- Cofficient de diffusion

- Teneur en eau volumique

- Vitesse de convection

- Fonction retard

- Rayon atomique et valence des ions

- Température

- Tortuosité du sol et taille des pores

- Taille des particules

- Nature minéralogique du sol et densité

- Taille des grains

- Naoure du milieu récepteur (sol, cours d'eau)

- Pression partielle 


\begin{tabular}{|c|c|c|c|}
\hline \multicolumn{2}{|c|}{ Terme considéré } & Facteurs d'influence & Paramètres de caractérisation \\
\hline Terme C & $\begin{array}{l}\text { Milieux } \\
\text { récepteurs } \\
\text { aquatiques (CA) }\end{array}$ & $\begin{array}{l}\text { - Type de milieu concerné (superficiel ou } \\
\text { souterrain) } \\
\text { - Type d'interface concemée (« terre/eau » ou } \\
\text { « eau superficielle/sédiment saturé en eau ») } \\
\text { - Voie de contamination concernée (lessivage } \\
\text { de surface, infiltration et percolation en } \\
\text { profondeur, échanges cours d'eau/nappe) }\end{array}$ & $\begin{array}{l}\text { - Paramètres physiques (granulométrie et perméabilité des sédiments, dynamique } \\
\text { hydrogéologique) } \\
\text { - Paramètres physico-chimiques ( } \mathrm{pH} \text {, température, conductivité, oxygène dissous, teneurs } \\
\text { en polluants,...) } \\
\text { - Paramètres biologiques (toxicité vis-à-vis d'espèces indicatrices, bioaccumulation, } \\
\text { abondance, composition faunistique, diversité faunistique) }\end{array}$ \\
\hline Terme C & $\begin{array}{l}\text { Milieux } \\
\text { récepteurs } \\
\text { « sol » (CB) }\end{array}$ & $\begin{array}{l}\text { - Type de fonctionnement du sol (sol de } \\
\text { type alluvial, calcimagnésique, brunifié, } \\
\text { lessivé, podzolisé, hydromorphe ou } \\
\text { halomorphe) } \\
\text { - Propriétés biologiques du sol (aspects } \\
\text { fonctionnels, populationnels et } \\
\text { démographique pour les micro- } \\
\text { organismes, la faune du sol et les végétaux) } \\
\text { - Nature des constituants du sol } \\
\text { (constituants minéraux et organiques) } \\
\text { - Propriétés physiques du sol (structure, } \\
\text { porosité, aération, mouvement de l'eau) } \\
\text { - Propriétés chimiques du sol (pH, } \\
\text { pouvoir tampon, capacité d'échange des } \\
\text { cations, taux de saturation) }\end{array}$ & $\begin{array}{l}\text { - Micro-organismes: } \\
\text { - nombre total d'organismes et biomasse microbienne } \\
\text { - nombre de micro-organismes assurant une fonction clé } \\
\text { - diversité } \\
\text { - activités (métabolites, enzymes, ARNm) } \\
\text { - Faune du sol: } \\
\text { - bioaccumulation dans certains organismes (indicateurs de bioaccumulation) } \\
\text { - intoxication d'organismes « alarmes » (indicateurs toxicologiques) } \\
\text { - structure des peuplements (indicateurs biologiques) } \\
\text { - Flore: } \\
\text { - biomasse aérienne et racinaire } \\
\text { - distribution des espèces végétales } \\
\text { - activités métaboliques. }\end{array}$ \\
\hline
\end{tabular}

Ce tableau, qui constitue une synthèse et une base de réflexion commune à l'ensemble des équipes qui participent au programme, sera régulièrement complété et actualisé au cours des deux prochaines années du programme. Son contenu et sa forme actuelle doivent donc être considérés comme provisoires

\section{Tableau 2 : Liste des sous-scénarios étudiés pour chacun des termes}

Terme A

\section{Nature des déchets}

Poreux granulaire

Poreux massif

Vitreux granulaire ou massif

Sous-scénario BI

Sous-scénario B2

Sous-scénario B3

Sous-scénario $n^{\circ} \mathrm{I}(\mathrm{A} \mid / \mathrm{BI})$

Sous-scénario $n^{\circ} 2(A \mid / B 2)$

Sous-scénario $n^{\circ} 3$ (A4/B3)

Scénario $n^{\circ} 4$ (A5/B3)

Scénario $n^{\circ} 5(A 5 / B I)$

\section{Nature du contact entre l'eau et les déchets}

Percolation
Sous-scénario Al

Lessivage

A2

A3

Terme B

Scénario de valorisation en sous-couche routière

Scénario de banalisation sous forme d'un tas disposé à la surface du sol Scénario de stockage (décharge)

\section{Terme $(\mathbf{A}+\mathbf{B})$}

Déchets poreux granulaires en sous-couche routière

Déchets poreux granulaires en tas

Déchets poreux granulaires stockés (avec possibilité d'immersion)

Déchets poreux massifs stockés (avec possibilité d'immersion)

Déchets poreux massifs valorisés comme matériau en contact avec de l'eau

\section{Nature du sol}

Sol grenu de perméabilité élevée $\left(K>10^{-5} \mathrm{~m} / \mathrm{s}\right)$

Sol fin de perméabilité moyenne $\left(10^{-8} \mathrm{~m} / \mathrm{s}<K<10-^{5} \mathrm{~m} / \mathrm{s}\right)$

Sol fin actif de perméabilité faible à très faible $\left(\mathrm{K}<10^{-8} \mathrm{~m} / \mathrm{s}\right)$

\section{Terme T}

$\begin{array}{cc}\text { Zone saturée } & \text { Zone non saturée } \\ \text { Sous-scénario TI } & \text { T4 } \\ \text { T2 } & \text { T5 } \\ \text { T3 } & \text { T6 }\end{array}$

Terme $C$ : Milieux récepteurs aquatiques (CA)

Type d'interface

Milieux

Interface solleau (berges de cours d'eau, Sous écoulement du cours d'eau zone non saturée/zone saturée) (eau superficielle/sédiment saturé en eau)

Milieu aquatique courant superficiel Milieu aquatique souterrain perméable Milieu aquatique souterrain colmaté
$\mathrm{C}_{A} 4$

$\mathrm{C}_{\mathrm{A}} 5$
$\mathrm{C}_{\mathrm{A}} 2$

$\mathrm{CA}_{\mathrm{A}} 3$ mmersion

A5

A6

\section{Facteurs et paramètres à} prendre en compte pour un scénario d'étude global donné

Tous les facteurs et paramètres qui figurent dans les colonnes du tableau $n^{\circ} I$ ne sont bien entendu pas à prendre en compte à chaque fois que l'on doit se prononcer sur l'écocompatibilité d'un dépôt de déchets. En effet, en fonction de la situation rencontrée (ou du scénario imaginé), seule une partie de ceux-ci sera à considérer. Par ailleurs, afin de ne pas multiplier inutilement des travaux identiques, un scénario global pourra être conçu comme la combinaison de "sous-scénarios " correspondant à chacun des termes de l'évaluation de l'écocompatibilité. Ces « sous-scénarios » peuvent être définis sur la base du ou des facteurs d'influence prépondérant pour le terme considéré. Leur liste est donnée dans le tableau $n^{\circ} 2$. La liste des facteurs et paramètres à prendre en compte pour chacun d'entre eux a ensuite été établie. Cette dernière n'est toutefois pas fournie ici en raison de sa longueur.

Pour l'étude d'un scénario global 


\section{Tableau 3 : Principaux facteurs et paramètres à prendre en compte}

\section{pour l'étude du scénario global $n^{\circ}$ I (AI, B2, T5, CAI et CB3)}

Principaux facteurs à prendre en compte

Principaux paramètres à prendre en compte

Facteurs liés au scénario

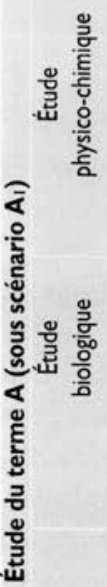

- Structure poreuse du déchet

- État granulaire du déchet

- Contact de l'eau avec le déchet par percolation essentiellement

- Mauvaise oxygénation sauf en surface surface

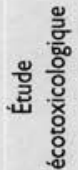

- Milieux aquatique courant superficiel. - Interface terre/eau

- Type de dépôt (tas) granulaires)

\section{- Circulation de l'eau en zone non saturé} - Sol de perméabilité moyenne.
- Pas de contact avec la lumière, sauf en

\section{Facteurs complémentaires}

- Conditions climatiques - Paramètres physico-chimiques: composition chimique et minéralogique, porosité, (pluviométrie, cycles de granulométrie, surface spécifique,

température, de gel/dégel et - Paramètres mécaniques, structurels et de vieillissement : résistance mécanique, d'humidification/séchage,...) taux de délitescence, sensibilité à la carbonatation, résistance au gel/dégel,

- Nature du terme B humidification/séchage

- Nature chimique de la solution - Paramètres de comportement à la lixiviation : contenu lixiviable, identification des de lixiviation

- Conditions d'oxygénation. paramètres du mécanisme principal de lixiviation, caractérisation du régime d'écoulement, sensibilité chimique aux conditions de lixiviation.

- Apports de nitrates et sulfates - Paramètres basés sur des analyses physico-chimiques ou biologiques : $\mathrm{CAE}, \mathrm{pH}$ - Apports de nutriments organiques et minéraux

- Contact avec un milieu micro biologiquement actif

- Paramètres ci-dessus de surface et/ou $\mathrm{pH}$ en suspension aqueuse, Eh en suspension aqueuse; pour lo couverture : soufre, sulfures, et autres formes réduites du soufre, fer (II) ; pour

lintérieur du tas : sulfates, nitrates, soufre

- Paramètres basés sur des tests de comportement : biodisponibilité des substances nutritives et énergétiques, présence de substances inhibitrices, biodégradabilité de la fraction organique

- Facteurs relatifs aux condi- - Effets globaux : survie, reproduction, croissance, comportement tions de l'essai (nature, durée - Effets spécifiques : altération du patrimoine génétique, et fréquence de l'exposition facteurs physiques, chimiques et microbiologiques susceptibles d'influer sur l'ècotoxicité des déchets)

- Facteurs relatifs aux organismes testés (type, nombre, âge, santé et stade de développement)

- Facteurs relatifs aux déchets testés (voir étude physica-chimique et biologique du terme $A$ )

- Conditions climatiques (plu- - Paramètres liés à la géométrie du tas (forme, pentes, surfaces)

viométrie, température, cycles - Paramètres liés à la structure du dépôt (nature, épaisseur et capacité de rétention de température, de gel/dégel et en eau de la terre végétale, résistance de celles-ci au cycle gel/dégel, d'humidification séchage...). humidification/séchage, type de végétation et capacité d'évaporation).

- Nature géologique, géotechni- - Paramètres liés à la mise en œuvre des déchets (état de surface, perméabilité, taux que et hydrogéologique du de compactage et vitesse de remplissage).

milieu récepteur

- Paramètres liés à la stabilité d'ensemble du tas (teneur en eau optimale, indice portan - Présence de végétaux et d'ani- immédiat, cohésion, angle de frottement). maux fouisseurs.

- Paramètres indispensables : coefficient de diffusion du soluté, conductivité hydraulique, teneur en eau volumique du sol.

- Paramètres supplémentaires : coefficient de diffusion de l'eau, teneur en eau initiale, courbe de rétention du sol.

Voies de contamination - Paramètres physiques (granulométrie des sédiments).

concernées (lessivage de - Paramètres physico-chimiques $(\mathrm{pH}$, température, conductivité, oxygène dissous, surface, infiltration et teneur en polluants,...).

percolation en profondeur, - Paramètres biologiques : étude des communautés biologiques d'invertébrés échanges cours d'eau/nappe). aquatiques (abondance, composition faunistique, diversité, présence/absence de certaines espèces).

Fonctionnement du sol du type « sols brunifiés ॥

- Propriétés biologiques du sol - Microorganismes:

(aspects fonctionnels et - nombre total d'organismes et biomasse microbienne,

démographiques pour les • nombre de microorganismes assurant une fonction clé,

microorganismes, la faune du - diversité,

sol et les végétaux) - activités (métabolites, enzymes, ARNm)

- Nature des constituants - Faune dusol:

organiques et minéraux du sol. - bioaccumulation dans certains organismes (indicateurs de bioaccumulation),

- Propriétés physiques du sol - intoxication d'organismes » alarmes » (indicateurs toxicologiques),

(structure, porosité, aération, • structure des peuplements (indicateurs biologiques)

mouvement de l'eau), - Végétation:

- Propriétés chimiques du sol - distribution des espèces végétales

( $\mathrm{pH}$, pouvoir tampon, capacité - biomasse aérienne et racinaire

d'échange des ions, taux de $\cdot$ activités métaboliques saturation). 
donné, il ne reste plus ensuite qu'à répertorier l'ensemble des facteurs et paramètres correspondant à la combinaison effectuée.

Afin d'illustrer la méthodologie adoptée, deux scénarios globaux, construits par combinaison de divers sous-scénarios, sont présentés dans les tableaux $n^{\circ} 3$ et 4 .

Scénario global $n^{\circ}$ I (Al, B2, T5, CAl et CB3) :

Dépôt de déchets poreux granulaires en tas à la surface d'un sol non saturé, de perméabilité moyenne, siège des milieux récepteurs " sol » caractéristiques des sols brunifiés et situé en amont d'un milieu récepteur aquatique de type " courant superficiel ».

Scénario global $n^{\circ} 2$ (A5, BI, T4, CAl et CB3) :

Valorisation de déchets poreux massifs dans un ouvrage contenant de l'eau, situé à la surface d'un sol perméable, non saturé, siège des milieux récepteurs « sol » caractéristiques des sols brunifiés, lui-même situé au-dessus d'un milieu récepteur aquatique souterrain perméable.

On notera dans ces tableaux que la liste des facteurs prépondérants, utilisés pour définir globalement les sous-scénarios, a été complétée par une liste de facteurs, dits « secondaires ॥, nécessaires pour une description et une étude plus détaillée de ceux-ci.

\section{POURSUITE DES TRAVAUX (DEUXIĖME ET TROISIÈME ANNÉES DU PROGRAMME)}

\section{Méthodologie globale}

Les principales informations à acquérir pour l'évaluation de l'écocompatibilité des déchets ont été présentées précé-

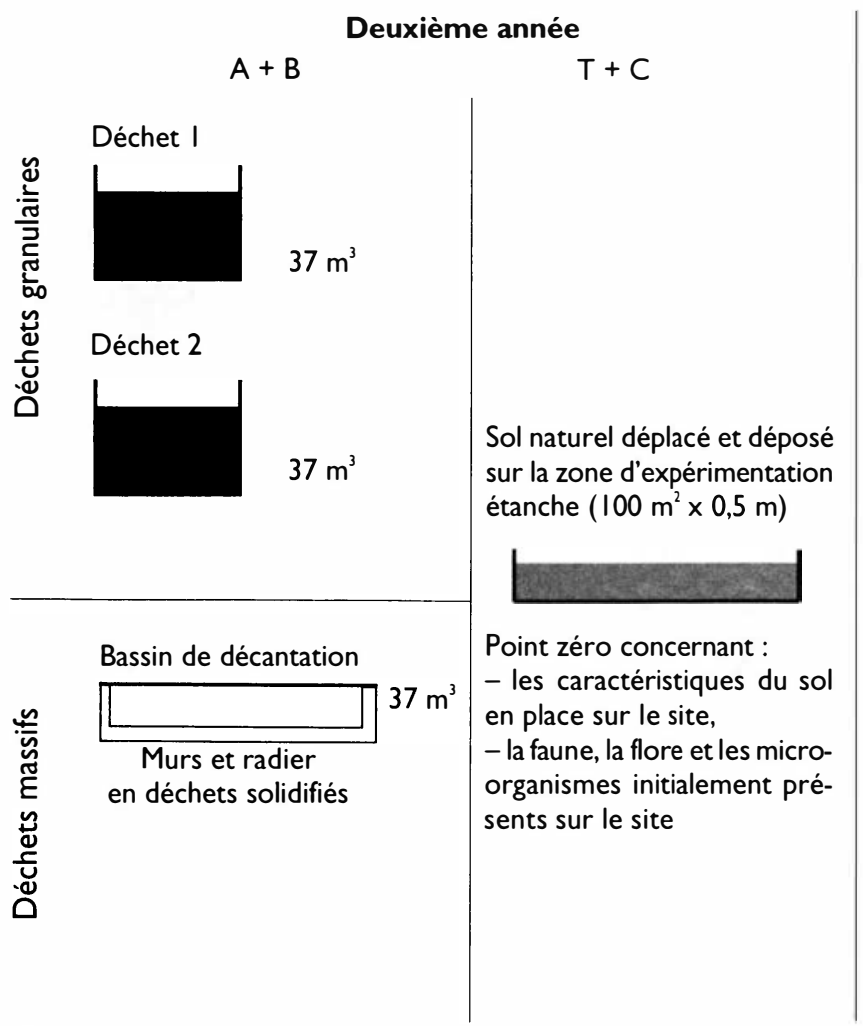

demment.

II reste maintenant à intégrer ces données au sein d'une méthodologie globale cohérente. C'est l'un des objectifs prioritaires de la deuxième année du programme.

\section{Validation expérimentale et calibration de la méthodologie proposée}

Afin d'évaluer les flux polluants émis par les déchets et les flux acceptables par les milieux récepteurs, les paramètres de caractérisation pertinents vont, dans un premier temps, être mesurés en laboratoire pour les deux scénarios globaux présentés ci-dessus, à savoir un scénario de banalisation et un scénario de valorisation.

Ceux-ci correspondent volontairement à des conditions d'exposition fortes permettant d'obtenir des flux de polluants importants qui, après modulations diverses, conduiront à des impacts mesurables et variables sur les milieux récepteurs. Les niveaux d'acceptation par les milieux récepteurs étant établis, il sera alors possible de se prononcer, pour un même déchet, sur l'écocompatibilité de scénarios à exposition plus faible (Centres de stockage réglementaires, par exemple). Parallèlement aux études de laboratoire, des expérimentations vont être lancées sur le site du Cered (voir détails sur la figure $n^{\circ} 3$ ) de manière à mesurer les mêmes flux à grande échelle (pilotes de 30 à $300 \mathrm{~m}^{3}$ ) et dans des conditions de terrain.

L'objectif de cette double expérimentation est de comparer les estimations de laboratoire avec les observations de terrain de manière à valider et à calibrer les outils de laboratoire sélectionnés.

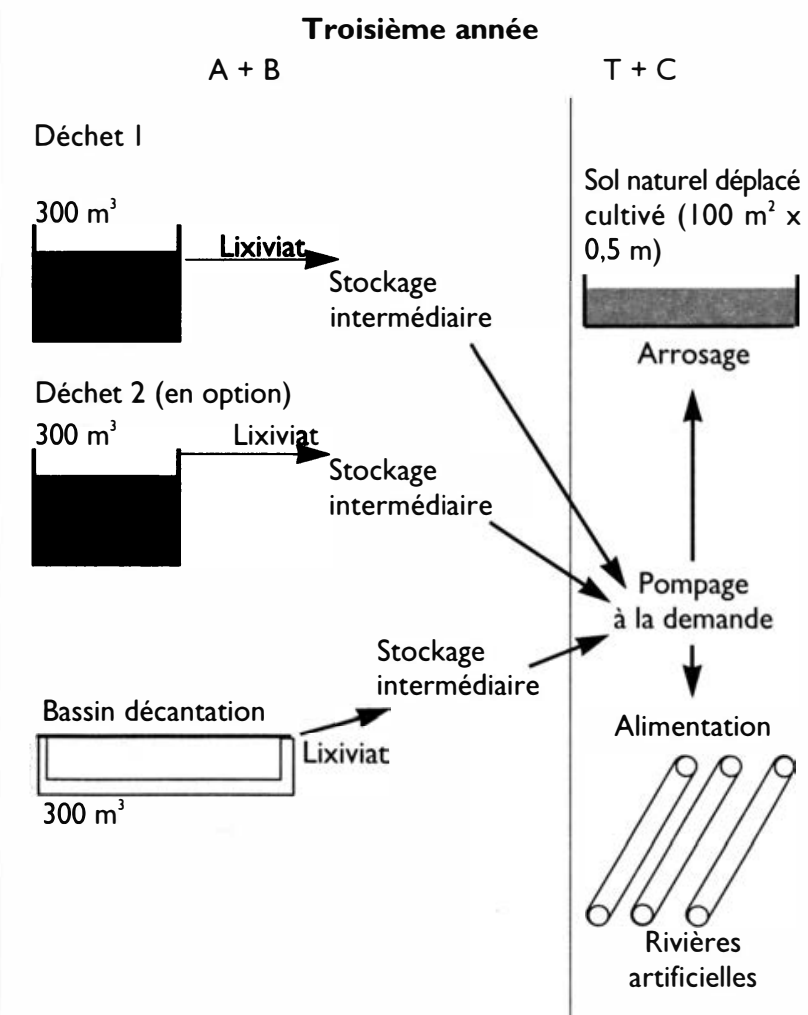

Figure 3 : Projet d'expérimentations à grande échelle pour les $2^{\text {ìme }}$ et $3^{\text {ìme }}$ années du programme 


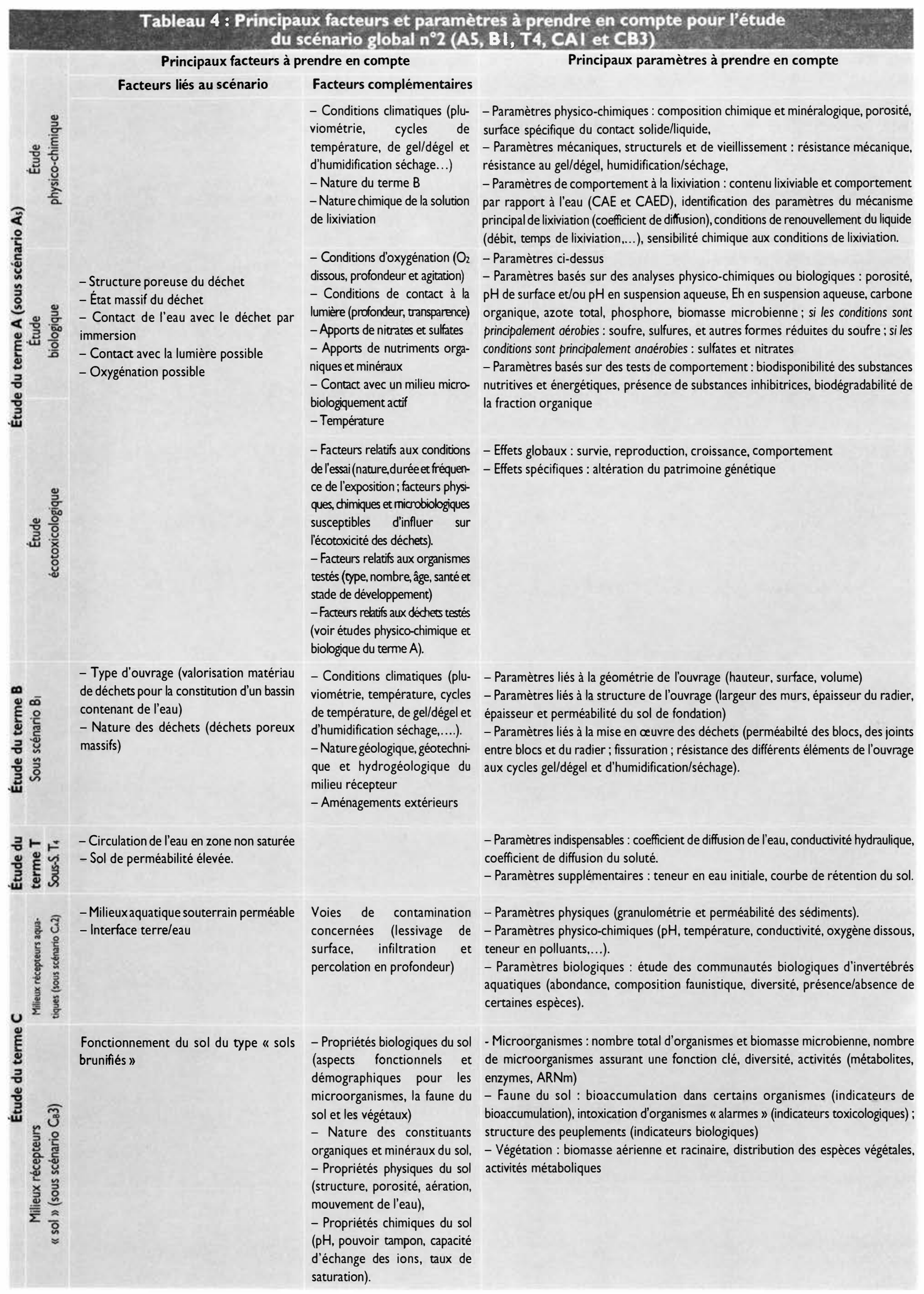




\section{CONCLUSION}

Ambitieux au niveau de l'objectif à atteindre et nécessitant le travail conjoint de nombreuses équipes scientifiques appartenant à des disciplines très différentes, le programme « Ecocompatibilité » n'a été lancé qu'après plusieurs années de réflexion, au sein de plusieurs équipes de l'Ademe d'abord, puis avec les chercheurs sélectionnés.

La première année d'étude, bibliographique et critique, a permis d'une part, de vérifier sa pertinence au regard des préoccupations du moment, et d'autre part, de dégager des priorités d'études sur des bases scientifiquement cohérentes.

Ce programme vient aujourd'hui d'être encouragé par le Comité consultatif de modernisation de la gestion des déchets qui a accepté de le financer à hauteur de $50 \%$. II devrait en effet permettre de définir avec clairvoyance les conditions optimums d'élimination et de valorisation des déchets, tant au niveau économique qu'au niveau de leur impact sur l'environnement.

A terme, l'écocompatibilité pourrait également s'avérer un outil de communication très utile au moment de l'ouverture d'un nouveau centre de stockage ou d'un chantier de valorisation.

\section{* Valérie Mayeux}

Ademe, Centre d'Angers - 2, square Lafayette - BP 406 - 49004 Angers cedex 01

\section{** Yves Perrodin}

Insa de Lyon, Division Polden, Bât. CEI - BP 2132 - 27, boulevard du II novembre $1918-69603$ Villeurbanne cedex

\section{Notes :}

I. Outre les deux auteurs, chargés respectivement du suivi et de la coordination du programme Ademe en cours sur l'écocompatibilité des déchets, le présent article est le fruit du travail de nombreuses personnes : Radu Barna (Polden-Insavalor), Pierre Moszkowicz, Rémy Gourdon et Eric Duhoo (Laepsi-Insa de Lyon), Gérard Didier, Véronique Norotte et Ibrahim Alimi (Laboratoire de GéotechniqueInsa de Lyon), Jean-François Ferard et Benoît Ferrari (CSE-Metz), Lucile Monrozier (Laboratoire d'Écologie Microbienne des SolsUniversité Lyon I), Jean-François Fruget et Sylvie Plenet (ARALEPBP), Christine Texier et Daniel Cluzeau (Station Biologique de Paimpont), Pierre Faivre (Laboratoire d'Écologie Végétale-Université de Savoie), Hervé Billard, Lucie Lambolez et Philippe Salmon (Cered).

2. Au sens de la loi, les déchets ultimes sont « des déchets résultant ou non du traitement des déchets, qui ne sont plus susceptibles d'être traités dans les conditions techniques et économiques du moment, notamment par extraction de la part valorisable ou par réduction de leur caractère polluant ou dangereux. De tels déchets sont essentiellement solides, minéraux, avec un potentiel polluant constitué de métaux lourds peu mobilisables. Ils sont très peu réactifs, très peu évolutifs et très peu solubles 1 . 